\title{
El papel de la autofagia en enfermedades pulmonares
}

\author{
Mariana Maciel-Herrerías, 凶Sandra Cabrera-Benítez
}

Universidad Nacional Autónoma de México, Ciudad de México.

Trabajo recibido: 27-IV-2016; aceptado: 05-VIII-2016

\begin{abstract}
RESUMEN. La autofagia es un proceso fundamental de degradación intracelular de organelos y proteínas dañadas. La autofagia es también un mecanismo esencial para la adaptación al estrés, la supervivencia y la homeostasis celular. Paradójicamente, la autofagia también puede promover la muerte celular, pero cuándo y cómo la autofagia puede tener funciones pro o antiapoptóticas, es aún desconocido. La autofagia involucra el reclutamiento y la degradación de organelos dañados, agregados de proteínas, proteínas de larga vida y patógenos en vesículas de doble membrana llamadas autofagosomas, que luego se fusionan con los lisosomas para formar los autofagolisosomas (o autolisosomas). Alteraciones en la autofagia han sido implicadas en una amplia gama de trastornos, incluyendo enfermedades pulmonares. En esta revisión, se discute lo que al presente se sabe sobre el papel de la autofagia en el inicio y la progresión de diferentes trastornos pulmonares. Algunos estudios indican que la autofagia podría tener un papel deletéreo en la patogénesis de la enfermedad pulmonar obstructiva crónica y del asma. En otros trastornos como la fibrosis pulmonar idiopática y la fibrosis quística, la inhibición de la autofagia puede contribuir a la patogénesis.
\end{abstract}

Palabras clave: Apoptosis, asma, autofagia, homeostasis, enfermedad pulmonar obstructiva crónica, fibrosis pulmonar idiopática, fibrosis quística.

ABSTRACT. Autophagy is a fundamental process of intracellular degradation of damaged organelles and proteins. Autophagy is also a crucial mechanism for adaptation to stress, survival and cellular homeostasis. Paradoxically, autophagy can also promote cell death, but when and how autophagy may have pro- or anti-apoptotic functions is still unknown. Autophagy involves the recruitment and degradation of damaged organelles, protein aggregates, long-lived proteins and pathogens, into double membrane vesicles called autophagosomes which then fuse with the lysosome to form autolysosomes. Alterations in autophagy have been implicated in a wide range of disorders including lung diseases. In this review, we discuss what is currently known about the role of autophagy in the initiation and progression of different pulmonary disorders. Some studies indicate that autophagy could have a deleterious role in the pathogenesis of chronic obstructive pulmonary disease and asthma. In other disorders, such as idiopathic pulmonary fibrosis and cystic fibrosis, impaired autophagy may contribute to pathogenesis.

Key words: Apoptosis, asthma, autophagy, homeostasis, chronic obstructive pulmonary disease, idiopathic pulmonary fibrosis, cystic fibrosis.

\section{INTRODUCCIÓN}

La principal función de los pulmones es el intercambio gaseoso. El pulmón no sólo es una barrera mecánica y física para impedir la entrada de partículas extrañas al organismo; sino también es la primera línea de defensa contra las agresiones ambientales y la infección debido a su capacidad para organizar, tanto la respuesta inmune innata como la respuesta adaptativa. Recién se ha descrito que la autofagia es un proceso celular esencial para mantener la integridad estructural y funcional del pulmón. ${ }^{1,2}$

Alteraciones en la actividad autofágica se han asociado a diversas patologías como el cáncer, enfermedades neurodegenerativas, infecciosas, autoinmunes, crónico-degenerativas e inflamatorias. ${ }^{3}$ Sin embargo, el estudio de la asociación de la autofagia con distintas enfermedades pulmonares inició hace pocos años. Hoy existen evidencias crecientes que muestran que la autofagia está vinculada a la patogénesis de distintos trastornos pulmonares como la fibrosis quística, el asma, la enfermedad pulmonar obstructiva crónica (EPOC) y la fibrosis pulmonar idiopática (FPI).

\section{AUTOFAGIA: UN MECANISMO DE DEGRADACIÓN INTRACELULAR}

La autofagia es un proceso de degradación intracelular que se caracteriza por la formación de vesículas de doble membrana denominadas autofagosomas, estas vesículas secuestran material citoplasmático y después se fusionan con el lisosoma (el cual contiene enzimas hidrolíticas), formando el autofagolisosoma o autolisosoma, en donde ocurre la degradación del material 
invaginado. Los aminoácidos y moléculas pequeñas que son generadas por autofagia, son devueltos al citoplasma para la generación de energía y para la síntesis de nuevas proteínas y biomoléculas. ${ }^{3,4}$ El principal inductor de la autofagia es el déficit de nutrientes, sin embargo, se ha demostrado que la activación de la autofagia es un mecanismo de supervivencia celular frente a diversas condiciones de estrés, incluyendo el estrés oxidativo, la inflamación, la agregación de proteínas, el estrés del retículo endoplásmico, el estrés metabólico, la presencia de patógenos y alteraciones de la función mitocondrial. ${ }^{4}$ La autofagia juega un papel importante en la homeostasis celular y tisular al contribuir en la generación de energía a partir de eventos de degradación, en el control de calidad de las proteínas y organelos, en la eliminación de proteínas de larga vida y patógenos, así como en la regulación de la muerte celular. ${ }^{5}$

Existen tres tipos de autofagia: la macroautofagia, la microautofagia y la autofagia mediada por chaperonas. Las diferencias en los mecanismos de reconocimiento y el tipo de degradación de los sustratos u organelos blanco es lo que caracteriza a cada una de estas variantes. ${ }^{4,5}$

La maquinaria central del proceso de autofagia está compuesta por más de 30 proteínas, entre ellas las denominadas Atg, siglas que provienen del inglés Autophagy. La vía de la autofagia procede a través de cinco fases: (1) la nucleación, que es la formación de una estructura de doble membrana o membrana de aislamiento, que también se denomina fagóforo, (2) la expansión de la membrana del fagóforo por la incorporación de la proteína LC3-II, (3) la maduración de esta estructura en el autofagosoma y el secuestro de material citoplásmico a degradar, (4) la fusión del autofagosoma con el lisosoma, lo que resulta en la formación de los autofagolisosomas o autolisosomas y, por último, (5) la degradación de los materiales biológicos secuestrados por las enzimas hidrolíticas del lisosoma y el reciclamiento de moléculas (ante todo aminoácidos, lípidos, azúcares y nucleótidos) (figura 1). $\cdot^{3-5}$

La nucleación y formación del fagóforo es iniciada por la cinasa de serina y treonina ULK1 (del inglés unc-51 like autophagy activating kinase 1). Una vez activado el complejo ULK1, éste activa al complejo Ptdlns3K (del inglés phosphatidylinositol 3-kinase) que incluye a miembros como Beclin 1, Atg14, Vps15, Vps34 y Ambra1. En la expansión de la membrana del fagóforo, hasta el cierre de la vesícula de doble membrana para formar el autofagosoma maduro, participan dos sistemas de conjugación tipo ubiquitina, que son Atg12-Atg5-Atg16 y Atg4-Atg7-Atg3/LC3-PE (fosfatidiletanolamina). ${ }^{5} \mathrm{Al}$ final, el autofagosoma se fusiona con el lisosoma, y el material secuestrado es degradado por las enzimas lisosomales (catepsinas, glucosidasas,

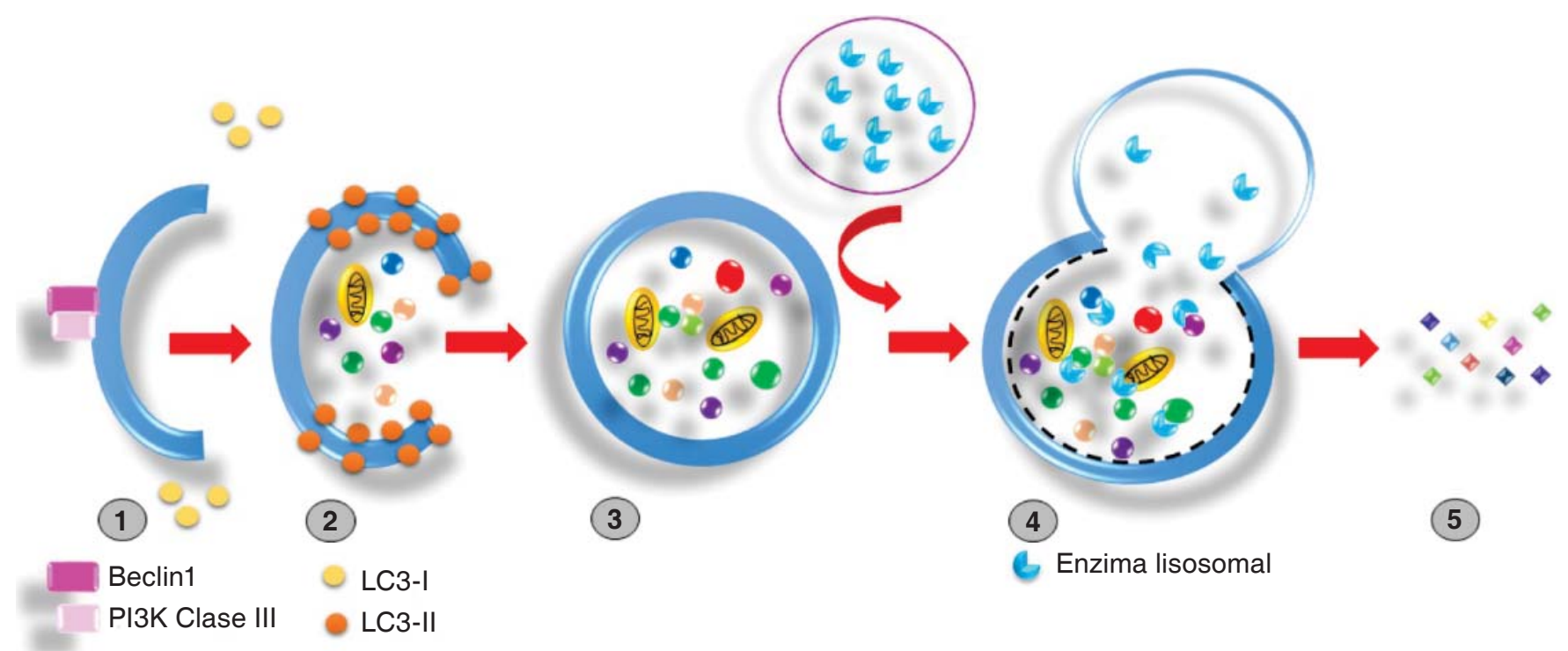

Figura 1. Etapas de la autofagia. En respuesta a distintos estímulos, se induce la formación de una membrana de aislamiento o fagóforo. 1) La nucleación de esta membrana requiere de la participación de los complejos de proteínas, Beclin-1 y la cinasa de clase III PI3K. 2) La incorporación de LC3-II a la membrana contribuye a la expansión del fagóforo. 3) Formación del autofagosoma maduro, el cual contiene el material citoplásmico. 4) El autofagosoma se fusiona con el lisosoma, que contiene enzimas hidrolíticas que degradarán el material secuestrado. 5) Los aminoácidos, azúcares, lípidos y nucleótidos generados por la degradación lisosomal son devueltos al citoplasma para ser reutilizados en la síntesis de nuevas biomoléculas. 
lipasas, sulfatasas). Los componentes de las biomoléculas degradadas, por ejemplo aminoácidos, son devueltos al citoplasma para la obtención de energía y para la síntesis de nuevas biomoléculas (Autophagy: renovation of cells and tissues). ${ }^{4,5}$

\section{AUTOFAGIA Y ENFERMEDADES PULMONARES}

La desregulación de la actividad autofágica recién ha sido reportada en varias patologías pulmonares. En algunos casos, la patogénesis está relacionada con una activación excesiva de la autofagia, mientras que en otros casos está asociada con una disminución en el flujo autofágico. A continuación se describen los cambios en la autofagia que se han reportado para distintas enfermedades pulmonares.

\section{AUTOFAGIA EN FIBROSIS QUÍSTICA}

La fibrosis quística (FQ) es una enfermedad autosómica recesiva monogénica que afecta a cerca de 70,000 personas en todo el mundo. Las manifestaciones clínicas de la enfermedad son causadas por defectos en la proteína transmembranal CFTR (del inglés cystic fibrosis transmembrane conductance regulator) de 1,480 aminoácidos, que funciona como un canal de cloro en la membrana apical de las células epiteliales. ${ }^{6}$ Alrededor de 2,000 mutaciones se han identificado en el gen CFTR, pero la más frecuente es la F508del-CFTR. Las mutaciones del CFTRT han sido agrupadas en seis clases diferentes de acuerdo con su impacto patológico, siendo las mutaciones de la clase I las más severas, y las de la clase VI las menos severas. ${ }^{7,8}$

La FQ es una enfermedad sistémica, aunque la gama de manifestaciones clínicas es altamente heterogénea en distintos órganos y en general incluyen la insuficiencia exocrina del páncreas, pancreatitis, aumento de electrolitos en el sudor e infertilidad masculina; sin embargo, la enfermedad pulmonar progresiva es la manifestación más prevalente, ésta resulta de la formación de un moco pegajoso, disminución en la eliminación mucociliar, con aumento de espesor del moco, inflamación crónica, colonización bacteriana persistente e intratable que causa infecciones pulmonares recurrentes, principalmente asociadas con Pseudomonas aeruginosa., 9,10 $^{10}$

Se ha reportado que la función defectuosa de CFTR induce la formación de especies reactivas de oxígeno (ROS) y la activación persistente de la transglutaminasa-2 (TG2), así como un aumento en los niveles de la misma. Los niveles elevados de TG2 se han observado en varias patologías humanas asociadas a defectos en la autofagia, incluyendo enfermedades neurodegenerativas tales como la enfermedad de Alzheimer, de Hun- tington, de Parkinson, así como en las enfermedades inflamatorias crónicas. ${ }^{10,11}$

La acumulación de proteínas mal plegadas y la acumulación de agregados han sido observadas en las vías respiratorias de los pacientes con $\mathrm{FQ}$, esto se ha asociado con defectos en la autofagia que resultan de una activación persistente de TG2 en las células epiteliales pulmonares. Además, en extractos de proteína de biopsias nasales de pacientes con FQ severa se ha demostrado la acumulación de p62 y bajos niveles de la forma conjugada de LC3 (LC3-II). Estos defectos en la autofagia resultan en la acumulación de proteínas dañadas y mal plegadas en las células epiteliales. ${ }^{11,12}$ Estudios recientes, han mostrado que la TG2 secuestra a la proteína Beclin-1 (BECN1), una proteína esencial para la autofagia, que junto con el complejo clase III de la fosfatidil-inositol 3 cinasa (PI3K) regulan la formación del agregosoma, así como la acumulación de la proteína p62 y acumulación de agregosomas. ${ }^{12,13}$

La restauración de Beclin-1 y la autofagia, ya sea mediante la sobreexpresión de Beclin-1 o por tratamiento con cistamina o antioxidantes, rescata la localización de Beclin-1, y revierte el fenotipo tanto in vitro, en las células epiteliales de biopsias nasales de humanos con $\mathrm{FQ}$, como in vivo en los ratones homocigotos mutantes CftrF508del. De forma interesante, el restablecimiento de la autofagia en los ratones homocigotos mutantes CftrF508del en el pulmón, resultó en la disminución de la respuesta inflamatoria y en una mejora en la función de las vías respiratorias. ${ }^{12-14}$

La autofagia también regula la eliminación de patógenos intracelulares. Estudios recientes han reportado que los macrófagos derivados de modelos murinos con FQ tienen una respuesta autofágica defectuosa frente a la infección bacteriana, lo que promueve la supervivencia de Burkholderia cenocepacia, así como la hipersecreción de la citocina proinflamatoria IL-1 $\beta$. $B$. cenocepacia es un patógeno que sobrevive de forma intracelular en los macrófagos y tiene la habilidad de replicarse en los mismos, causando tasas elevadas de morbilidad y mortalidad en pacientes con FQ. ${ }^{15}$ Una investigación reciente mostró in vitro, que el tratamiento con IFN $\gamma$ en macrófagos humanos derivados del pulmón de pacientes con $\mathrm{FQ}$, aumentó la actividad autofágica, la formación de autofagosomas y el tráfico lisosomal, resultando en una mejor eliminación de $B$. cenocepacia y en una disminución de la producción de citocinas inflamatorias. Sin embargo, más estudios son necesarios para determinar la eficacia del IFN $\gamma$ para el tratamiento de FQ no sólo en cultivo celular, sino también in vivo. ${ }^{16}$

Pseudomonas aeruginosa es un patógeno bacteriano oportunista y es considerado la principal causa de morbilidad y mortalidad entre los pacientes con FQ. 
Aunque $P$. aeruginosa es un patógeno extracelular, informes recientes han demostrado que durante el curso de la infección, la bacteria adquiere la capacidad de entrar y residir dentro de células huésped, evadiendo de esta forma al sistema inmune. También se demostró que la inhibición de la vía de la autofagia afecta de manera significativa la eliminación de $P$. aeruginosa en los mastocitos y en las células epiteliales bronquiales humanas, mientras que la inducción del proceso promueve la muerte de bacterias, y la disminución de la carga bacteriana, la respuesta inflamatoria y el infiltrado neutrofílico in vivo. ${ }^{17,18}$ En este contexto, la activación de la autofagia con inductores, podría ser un tratamiento útil para combatir las infecciones oportunistas en la FQ.

Paradójicamente, se ha descrito que Staphylococcus aureus (otra bacteria oportunista asociada con FQ) induce la formación de autofagosomas donde evita la fusión lisosomal para establecer un nicho replicativo y así escapar de la vía fagocítica. Se ha propuesto que el envolvimiento de bacterias en vesículas positivas para LC3 es independiente de Beclin1 y PI3K-Clase 3 , y que podría ser a través de una vía de la autofagia no canónica, inducida por la toxina $\alpha$-hemolisina que regula los niveles intracelulares de cAMP. Sin embargo, el papel específico de la autofagia en infecciones de S. aureus asociadas con FQ se desconocen aún. ${ }^{18,19}$

En conjunto, las evidencias citadas previamente asocian el defecto del canal CFTR con una deficiencia en la actividad autofágica, y sugieren que la inducción y la activación de la autofagia podría constituir un enfoque diferente para la generación de nuevos fármacos para el tratamiento de la FQ (figura 2).

\section{AUTOFAGIA EN LA ENFERMEDAD PULMONAR OBSTRUCTIVA CRÓNICA}

La enfermedad pulmonar obstructiva crónica (EPOC) se caracteriza por presentar bronquitis asociada con inflamación de las vías aéreas y obstrucción por moco, así como enfisema caracterizado por pérdida de la superficie alveolar. La EPOC representa la cuarta causa de muerte mundial y el principal factor de riesgo es la inhalación de humo de cigarro; aunque la exposición a diversas partículas como los gases generados por la quema de combustibles o de madera, así como la deficiencia genética de la proteína alfa 1-antitripsina, también son factores de riesgo para el desarrollo de EPOC. ${ }^{20,21}$ Las células epiteliales son las más susceptibles al daño por el humo de cigarro, éste ocasiona inflamación, ${ }^{22}$ estrés oxidativo, ${ }^{23}$ apoptosis, ${ }^{24,25}$ alteraciones en la proteostasis, estrés de retículo endoplásmico, disminución del sistema ubiquitina-proteasoma, ${ }^{26}$ así como un defecto en el balance de las actividades de proteasas y antiproteasas. ${ }^{27}$

Se ha reportado que en biopsias de pacientes con EPOC en distintas etapas de la enfermedad, hay un incremento en los niveles de autofagia comparados con pulmones de pacientes sanos. Asimismo, ha sido demostrado en modelos in vivo e in vitro, que la exposición al humo de cigarro conlleva a la activación de

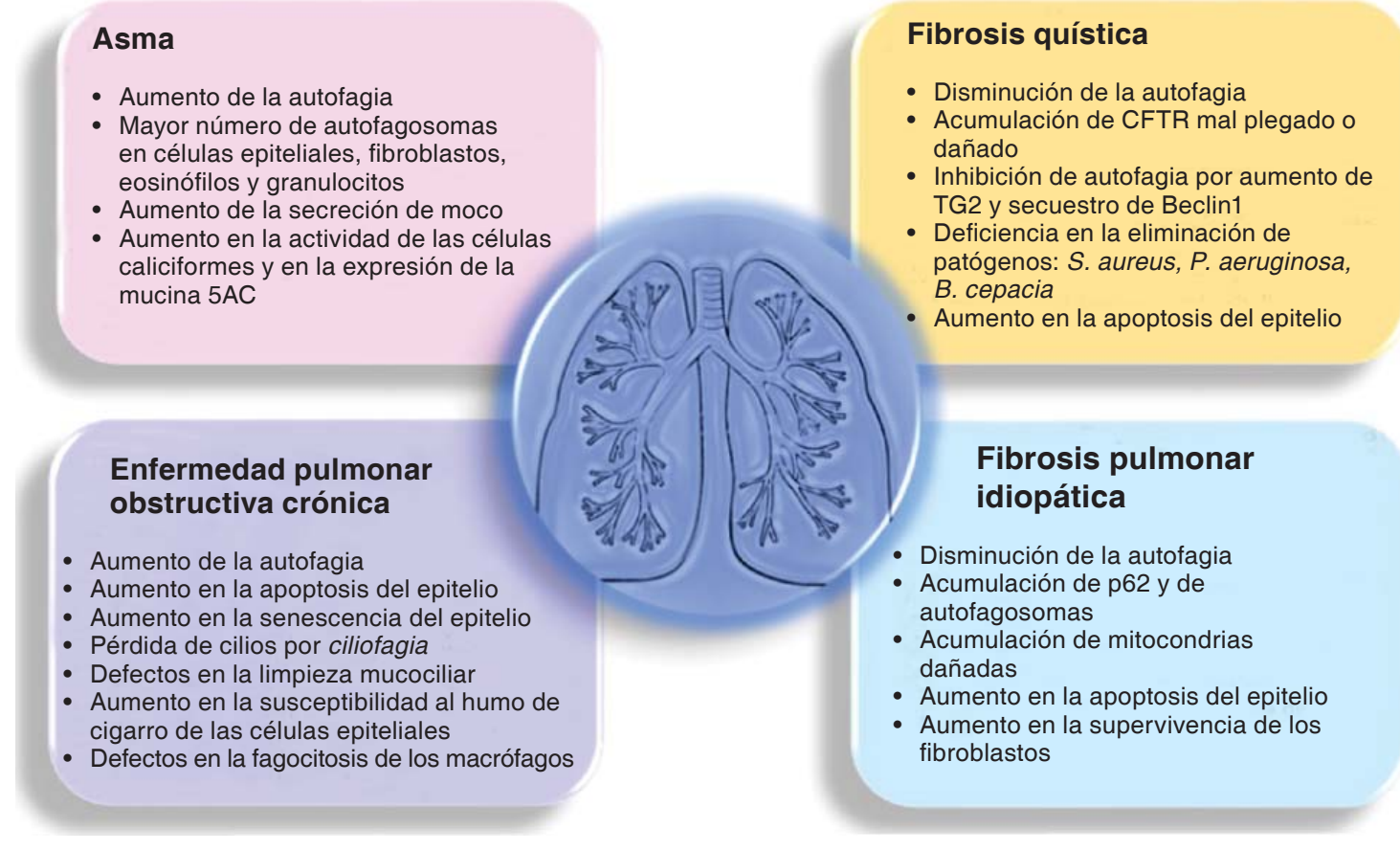

Figura 2.

Autofagia y enfermedades pulmonares. La correcta activación de la autofagia es esencial para mantener el funcionamiento e integridad del pulmón. En la figura se muestran alteraciones en la autofagia en diversas patologías pulmonares. 
la autofagia y la inducción de apoptosis en las células epiteliales. ${ }^{28}$ No obstante, a pesar de que la autofagia es un mecanismo de protección frente al estrés, que promueve la sobrevivencia de las células, se ha observado que in vitro la inhibición de proteínas esenciales de la autofagia (como LC3B o Beclin-1) disminuye la apoptosis y la citotoxicidad del humo de cigarro en células epiteliales. ${ }^{29}$ Se ha propuesto que la autofagia se activa en etapas tempranas de la enfermedad, mientras que la apoptosis sólo ocurre en etapas tardías donde la enfermedad es más severa. Esto sugiere que la activación temprana de la autofagia representa un mecanismo de protección para atenuar el daño inducido por la toxicidad del humo de cigarro; sin embargo, en etapas tardías, el incremento en la actividad autofágica podría promover la muerte celular en lugar de su supervivencia.

Con el uso de modelos animales experimentales de EPOC, realizados en murinos se ha demostrado que un ratón modificado genéticamente, deficiente de la proteína autofágica LC3B, está protegido de la remodelación pulmonar inducida por la exposición al humo de cigarro, y el tejido pulmonar de estos ratones mutantes no muestra un incremento en los marcadores apoptóticos al comparar con el pulmón del ratón silvestre. Una posible explicación de la disminución en la apoptosis es que el humo de cigarro podría estar promoviendo una actividad proapoptótica de LC3B a través de la interacción LC3B-Fas y, de esta manera, activar la vía extrínseca de la apoptosis. ${ }^{30}$

Por otro lado, una de las características que también se presentan durante la EPOC, es la acumulación de proteínas ubiquitinadas. La inhalación del humo de cigarro induce alteraciones en la proteostasis lo que conlleva a la formación de agregosomas que contribuyen a la patogénesis de la enfermedad. A pesar de que se ha descrito que hay un incremento en la autofagia y que es principalmente activada por el humo de cigarro, se ha visto en células epiteliales que la formación y acumulación de agregosomas no son eliminados debido a alteraciones en la autofagia; por el contrario, si se utiliza un inductor de la autofagia como la carbamazepina, se promueve la eliminación de los agregosomas y su acumulación disminuye significativamente. También se observó en un modelo in vivo de exposición crónica a humo de cigarro, que el tratamiento con carbamazepina inhibe el alargamiento de los espacios alveolares así como la activación de caspasas. ${ }^{31}$

Como ya se mencionó, en el pulmón existen barreras de defensa para proteger al pulmón de partículas y patógenos inhalados. Una de estas defensas la constituyen los macrófagos alveolares. Se ha visto que los macrófagos de pacientes fumadores y con EPOC, tienen un incremento en el número de autofagosomas; sin embargo, hay un defecto en la degradación vía autofágica, pues los autofagosomas no se fusionan con los lisosomas, lo que conlleva a su acumulación, además, existe una acumulación de mitocondrias dañadas y de proteínas ubiquitinadas afectando las funciones fagocíticas de los macrófagos. ${ }^{32}$ Se ha visto que macrófagos de pacientes con EPOC no eliminan correctamente a bacterias como Haemophillus influenzae y Streptococcus pneumoniae, ${ }^{33}$ por lo cual es probable que defectos en la autofagia en los macrófagos alveolares estén asociados con la alteración en la fagocitosis de los macrófagos, y contribuyan en la susceptibilidad al desarrollo de infecciones pulmonares oportunistas en pacientes con EPOC.

La barrera mucociliar constituye otro mecanismo de eliminación de partículas que quedan atrapadas en el moco de las vías aéreas. La exposición al humo de cigarro perturba esta función y conduce a defectos en la eliminación de estas partículas. La alteración en la función de las células ciliadas se debe a que hay un acortamiento en la longitud de los cilios de las células epiteliales, así como apoptosis de estas células y una posterior reepitelización por células caliciformes, lo que resulta en una producción de moco excesiva. Recién se propuso que el acortamiento de los cilios es debido a que eran reabsorbidos a través de la maquinaria autofágica por un mecanismo específico denominado «ciliofagia». ${ }^{34}$ En modelos in vitro, se observó que la exposición al humo de cigarro ocasiona que las células epiteliales ciliadas pierdan su integridad y viabilidad al ocurrir acortamiento y pérdida de los cilios. Asimismo, se ha demostrado que las células epiteliales ciliadas derivadas de un ratón modificado genéticamente, heterocigoto para la proteína autofágica Beclin-1, están protegidas de la ciliofagia (acortamiento de los cilios), inducida por el humo de cigarro en comparación con el ratón silvestre. ${ }^{35}$ Se ha sugerido que, quizás, el exceso de autofagia inducida por el humo de cigarro podría estar asociada con la degradación y pérdida de los cilios, alterando la viabilidad y funciones de las células epiteliales ciliadas.

Se sabe que diversos componentes de la autofagia pueden inducir la apoptosis. ${ }^{36}$ En un estudio se demostró que la exposición al humo de cigarro activa la vía extrínseca de la apoptosis a través de la formación del DISC (del inglés death inducing signaling complex) esencial para activar a las caspasas $8,3,6$ y 7 . La inhibición de la autofagia por medio del silenciamiento de Beclin-1 y LC3B en células epiteliales disminuye la formación del DISC y la activación de las caspasas 8 y $3 .{ }^{29}$

En conclusión, los niveles de autofagia se encuentran elevados en EPOC. A pesar de ser un proceso que 
precede a la apoptosis y que al parecer está asociado con la atenuación del daño, en pacientes con EPOC se ha descrito que hay una activación «exagerada» de la autofagia, lo que resulta perjudicial para las células al promover procesos como la apoptosis o la degradación de componentes estructurales esenciales de las células epiteliales como los cilios. Por otro lado, existen diferencias en la actividad autofágica, dependiendo del contexto celular, ya que en los macrófagos alveolares hay una deficiencia en el flujo autofágico y se ha postulado que esto podría contribuir a una mayor susceptibilidad a infecciones (figura 2).

\section{AUTOFAGIA EN ASMA}

El asma afecta a unos 300 millones de personas en todo el mundo. El asma es un trastorno fenotípicamente heterogéneo, en los últimos años se han descrito muchos subtipos clínicos diferentes de asma. En los niños se han descrito tres fenotipos de asma: sibilancia transitoria infantil, sibilancia no atópica del niño y sibilancia mediada por IgE. En los adultos el asma persistente de la infancia debe ser distinguida del asma que comienza en la edad adulta. Los fenotipos de asma del adulto son poco definidos aún y hasta ahora, la clasificación fenotípica se ha basado sobre todo en los factores etiológicos (por ejemplo, sensibilidad a la aspirina, infecciones respiratorias persistentes, factores ocupacionales, o exposición a sustancias tóxicas) o las características clínicas de la enfermedad (por ejemplo, leve, severa, moderada, o resistente a esteroides). ${ }^{37}$

El asma alérgica es una enfermedad crónica inflamatoria de tipo Th2 de las vías aéreas caracterizada por la obstrucción reversible al flujo aéreo y la hiperreactividad de las vías respiratorias. El asma presenta cambios estructurales en las vías aéreas respiratorias que incluyen fibrosis subepitelial, hiperplasia de células caliciformes, engrosamiento del músculo liso y aumento de la vascularización, estos cambios en conjunto son conocidos como la remodelación de la vía aérea. ${ }^{38}$

La asociación genética de la autofagia y el asma se ha evaluado en dos estudios recientes donde se exploraron variantes alélicas en genes Atg. En el primer estudio se analizó una población de 312 niños asmáticos de raza blanca/caucásica, y se encontró un polimorfismo de un solo nucleótido (SNP rs510432) en el gen ATG5 que podría estar relacionado con el asma infantil. Se reportó que esta variante alélica está asociada con la enfermedad y le confiere una mayor actividad al promotor de ATG5. La población de niños con asma evaluada en este estudio pertenece a la cohorte GCPCR (Greater Cincinnati Pediatric Clinic Repository) y el diagnóstico de asma fue realizado de acuerdo a la guía de la Sociedad Americana de Tórax (American Thoracic Society). ${ }^{39}$ El gen ATG5 está situado en la región cromosómica 6q21 y es de casi 141 pares de kilobases de longitud. En el segundo estudio se evaluó una población de 1,338 individuos caucásicos no hispánicos, y se identificó un SNP en el intrón 3 de ATG5 asociado con asma en adultos, localizado $7 \mathrm{~kb}$ río abajo del exón 3 y $8 \mathrm{~kb}$ río arriba del exón (SNP rs12212740 G>A); aunque, la consecuencia funcional de este SNP no se ha determinado. ${ }^{40}$

Además, por microscopia electrónica se evaluó el número de autofagosomas en fibroblastos y células epiteliales derivados de tejido de biopsia bronquial de un paciente asmático moderadamente grave, y se observó que estas vesículas de doble membrana fueron más prevalentes en las células derivadas del paciente con asma en comparación con las células derivadas de un sujeto sano. ${ }^{40}$

Utilizando un modelo de ratón para estudiar el asma, en donde se estimuló a los animales con IL33, se observó un aumento en la formación de las células caliciformes y un aumento en la secreción de mucina MUC5AC (mucina 5AC), proteína esencial en la formación del moco. Resulta interesante que en los ratones modificados genéticamente, deficientes de ATG16L1 (un gen esencial de la maquinaria autofágica), tratados con IL-33 se atenuó la formación de células caliciformes y la secreción de moco, en comparación con los controles de tipo silvestre (WT). ${ }^{41}$

Hace poco se ha propuesto que la secreción de mucina está asociada con una mayor formación de la proteína LC3-II y, por lo tanto, con una mayor actividad autofágica. En correlación con esto, la eliminación de la expresión de Atg5 o Atg14 en células epiteliales derivadas de tráquea de humano, tratadas con IL13, resultó en la hipertrofia de las células caliciformes y en una disminución en la secreción de MUC5AC y en la generación de ROS mediada por IL13. Estos datos sugieren que la inhibición de la autofagia podría atenuar el desarrollo de asma. ${ }^{41}$

En otro estudio, utilizaron una sonda fluorescente para examinar en número de autofagosomas en las células de sangre periférica y en eosinófilos y granulocitos de esputo derivado de pacientes con asma grave y no grave, se observó la acumulación de puntos fluorescentes en las células provenientes de pacientes con asma grave, en comparación con pacientes con asma no grave y con los controles sanos, lo cual indica que hay un mayor número de autofagosomas y esto podría estar asociado con el grado de severidad de la enfermedad. ${ }^{42}$

Estos datos parecen indicar que hay una correlación positiva entre autofagia y la severidad del asma; empe- 
ro, la acumulación de autofagosomas o un incremento en el número de éstos, no necesariamente indica un aumento en la actividad autofágica, sino que podría reflejar una deficiencia en la fusión de los autofagosomas con los lisosomas y defectos en la degradación lisosomal de los materiales intracelulares (figura 2). ${ }^{42}$

\section{AUTOFAGIA EN FIBROSIS PULMONAR IDIOPÁTICA}

La fibrosis pulmonar idiopática (FPI) es una enfermedad de etiología desconocida, es la patología más común de las neumopatías intersticiales idiopáticas y la más agresiva de las enfermedades intersticiales del pulmón. Es crónica, progresiva y letal y no tiene cura. Los pacientes que padecen FPI presentan insuficiencia respiratoria debido a la acumulación de fibras de colágena en los espacios alveolares. ${ }^{43} \mathrm{La}$ FPI es una enfermedad asociada con el envejecimiento ya que es más frecuente en adultos mayores de 60 años. Se ha propuesto que la FPI es el resultado de un envejecimiento prematuro en el pulmón caracterizado por la senescencia de las células epiteliales y por un proceso de reparación aberrante que conlleva a la acumulación exagerada de matriz extracelular. ${ }^{44,45}$

Se sabe que durante el envejecimiento y la FPI, hay una disminución de la actividad autofágica y esto se ha asociado con la acumulación de organelos y macromoléculas dañadas que contribuyen al desarrollo de distintas enfermedades. ${ }^{46}$

Al igual que en EPOC, en la FPI también se presenta un incremento en los niveles de distintos tipos de estrés que están ejerciendo daño a las células, tales como el estrés oxidativo y el estrés de retículo endoplásmico, ambos inductores de la autofagia. A pesar de estos estímulos, la actividad autofágica se encuentra severamente disminuida en el pulmón de pacientes con FPI. ${ }^{47}$ Asimismo, en pulmones con FPI, por medio de inmunohistoquímica se observó la acumulación de proteínas ubiquitinadas y del marcador de autofagia p62, en especial en zonas fibróticas. Al igual que en estas regiones, no se detectaba la presencia de LC3 y Beclin1 en comparación con zonas en donde no había daño y en zonas equivalentes en pulmones de pacientes sanos. ${ }^{48}$ Esto sugiere que en la FPI hay agregados de proteínas que se están acumulando en el pulmón y no están siendo degradados debido a una disminución en la autofagia, lo cual podría sensibilizar a las células frente a distintos tipos de estrés.

Se ha demostrado que en células epiteliales y en focos de fibroblastos de pulmón de pacientes con FPI, así como en el modelo animal de fibrosis pulmonar inducida por bleomicina, hay una activación aberrante de la cinasa mTOR, el principal regulador negativo de la autofagia. La inhibición farmacológica de mTOR a través de la rapamicina induce la activación de la autofagia y protege a los ratones del daño pulmonar inducido por la bleomicina. ${ }^{49}$ Estos datos sugieren que es probable que la deficiencia de la autofagia en las células epiteliales esté asociada con una incapacidad de las mismas a responder y atenuar los distintos tipos de estrés que se dan durante la enfermedad, haciéndolas más susceptibles al daño y a la muerte celular.

Por el contrario, en fibroblastos derivados de pacientes con FPI parece ser que la disminución de la autofagia promueve su supervivencia. Durante la FPI, incrementa la rigidez de la matriz extracelular y cambia su composición esto induce, a su vez, cambios en la expresión génica de los fibroblastos, promoviendo un fenotipo profibrótico. ${ }^{50}$ Se ha visto que cultivando fibroblastos provenientes de pulmón de pacientes con FPI en matrices rígidas, la autofagia es inhibida a través de la activación aberrante de mTOR. Los fibroblastos de pacientes control son capaces de reconocer el cambio de rigidez de la matriz, traduciéndolo como un estrés e induciendo la autofagia, la cual actúa como un mecanismo que sensibiliza a las células ante los cambios que ocurren en su ambiente extracelular, haciéndolas más susceptibles a la muerte celular. Sin embargo, los fibroblastos de FPI se «desensibilizan» ante este estrés a través de la inhibición de la autofagia y, por lo tanto, son más resistentes a la apoptosis inducida por una matriz rígida de colágena tipo I. ${ }^{51}$ Los resultados de estos estudios indican que la autofagia regula distintos procesos dependiendo no sólo del tipo celular, sino también del ambiente extracelular.

EI TGF- $\beta 1$ (del inglés transforming growth factor $\beta 1$ ) se considera que es una molécula profibrosante que estimula la diferenciación de fibroblastos a miofibroblastos exacerbando el depósito de colágena. ${ }^{52}$ En un estudio se observó que el TGF- $\beta 1$ inhibía la autofagia durante la diferenciación de los miofibroblastos y disminuía la expresión de genes de la maquinaria autofágica como ATG7 y ATG16L1 y PINK1 (del inglés PTEN-induced putative kinase 1, esta última proteína está involucrada en la degradación de las mitocondrias dañadas por medio de un proceso selectivo de autofagia denominado mitofagia). ${ }^{53}$ También se ha visto que en pulmones con FPI la expresión de PINK1 está disminuida, lo que conlleva a que la integridad de las mitocondrias se vea afectada y se acumulen mitocondrias dañadas; esto resulta en un incremento de la apoptosis en las células epiteliales promoviendo el desarrollo de la fibrosis pulmonar. ${ }^{54}$ En resumen, estos resultados indican que el TGF- $\beta 1$, al inhibir la actividad autofágica también altera la eliminación de mitocondrias dañadas contribuyendo con ello a la patogénesis de la enfermedad. 
Recién se estudió in vivo el papel de la autofagia en el modelo de fibrosis pulmonar inducida por bleomicina en el ratón deficiente del gen Atg4B (una cisteín-proteasa esencial para la formación de los autofagosomas). Se observó que el número de células apoptóticas era significativamente mayor $(60.03 \pm 2.4 \%$ en el pulmón del ratón mutante vs. $27.3 \pm 1.1 \%$ en el pulmón del ratón silvestre), además, por inmunohistoquímica usando un anticuerpo específico contra caspasa 3 activa, se determinó que la mayoría de las células apoptóticas eran células del epitelio alveolar y bronquiolar. Asociado con esto, la respuesta fibrosante, la extensión del daño y la acumulación de colágena, fue de forma significativamente mayor en los ratones deficientes de Atg4B. Los resultados de esta investigación indican que la proteína autofágica ATG4B es importante para mantener la homeostasis del epitelio pulmonar y para disminuir la respuesta inflamatoria y fibrosante inducidas por la bleomicina. ${ }^{55}$

En resumen, se ha demostrado in vivo e in vitro que la autofagia se encuentra disminuida en la FPI, y que alteraciones en este proceso contribuyen a variaciones en distintas poblaciones celulares para responder frente al estrés y eliminar moléculas dañadas que en conjunto contribuyen a la patogénesis de la FPI (figura 2).

\section{CONCLUSIÓN}

La autofagia es un mecanismo de respuesta ante diversos tipos de estrés y, por tanto, promueve la supervivencia en las células. La insuficiencia de la autofagia así como una hiperactivación crónica de este proceso tiene un impacto en la inducción y la progresión de enfermedades pulmonares. Es importante aclarar que la vía de la autofagia también se relaciona con la activación de la apoptosis. Como se mencionó previamente, dependiendo del tipo de daño, del tipo celular, así como del contexto en el que se encuentren las células, la activación o inhibición de la autofagia funcionará como un mecanismo de atenuación del daño y supervivencia o por el contrario promoverá la muerte celular exacerbando el daño. Es decir, es necesario comprender mejor el papel específico de la autofagia en cada tipo celular, cómo se modula el flujo autofágico y cómo podría estar influyendo en la patogénesis de las enfermedades pulmonares. El estudio del papel de la autofagia en las enfermedades pulmonares es emergente, muchas evidencias se están obteniendo a partir del análisis en modelos animales modificados genéticamente con deficiencia en genes esenciales de la maquinaria de la autofagia. Esto contribuirá a la comprensión de las múltiples vías de autofagia y su impacto en la patogénesis de las distintas enfermedades pulmonares, y podrá facilitar el diseño de nuevos inductores o inhibidores farmacológicos de la autofagia para desarrollar terapias más específicas.

\section{REFERENCIAS}

1. Rackley $C R$, Stripp BR. Building and maintaining the epithelium of the lung. J Clin Invest 2012;122(8):27242730. doi: 10.1172/JCI60519.

2. Whitsett JA, Alenghat T. Respiratory epithelial cells orchestrate pulmonary innate immunity. Nat Immunol 2015;16(1):27-35. doi: 10.1038/ni.3045.

3. Mizushima N, Levine B, Cuervo AM, Klionsky DJ. Autophagy fights disease through cellular self-digestion. Nature 2008;451(7182):1069-1075. doi: 10.1038/nature06639.

4. Wong E, Cuervo AM. Integration of clearance mechanisms: the proteasome and autophagy. Cold Spring Harb Perspect Biol 2010;2(12):a006734. doi: 10.1101/ cshperspect.a006734.

5. Mizushima N, Yoshimori T, Ohsumi Y. The role of Atg proteins in autophagosome formation. Annu Rev Cell Dev Biol 2011;27:107-132. doi: 10.1146/annurevcellbio-092910-154005.

6. Quon BS, Rowe SM. New and emerging targeted therapies for cystic fibrosis. BMJ 2016;352:i859. doi: 10.1136/ bmj.i859.

7. O'Sullivan BP, Freedman SD. Cystic fibrosis. Lancet 2009;373(9678):1891-1904. doi: 10.1016/S01406736(09)60327-5.

8. De Boeck K, Zolin A, Cuppens H, Olesen HV, Viviani $\mathrm{L}$. The relative frequency of CFTR mutation classes in European patients with cystic fibrosis. J Cyst Fibros 2014;13(4):403-409. doi: 10.1016/j.jcf.2013.12.003.

9. Okumura MJ, Kleinhenz ME. Cystic fibrosis transitions of care: lessons learned and future directions for cystic fibrosis. Clin Chest Med 2016;37(1):119-126. doi: 10.1016/j. ccm.2015.11.007.

10. Esposito S, Tosco A, Villella VR, Raia V, Kroemer G, Maiuri L. Manipulating proteostasis to repair the F508del-CFTR defect in cystic fibrosis. Mol Cell Pediatr 2016; 3(1):13. doi: 10.1186/s40348-016-0040-z.

11. De Stefano D, Villella VR, Esposito $S$, et al. Restoration of CFTR function in patients with cystic fibrosis carrying the F508del-CFTR mutation. Autophagy 2014;10(11):20532074. doi: 10.4161/15548627.2014.973737.

12. Luciani A, Villella VR, Esposito $S$, et al. Targeting autophagy as a novel strategy for facilitating the therapeutic action of potentiators on $\Delta F 508$ cystic fibrosis transmembrane conductance regulator. Autophagy 2012;8(11):1657-1672. doi: 10.4161/auto.21483.

13. Luciani A, Villella VR, Esposito $S$, et al. Defective CFTR induces aggresome formation and lung inflammation in cystic fibrosis through ROS-mediated autophagy inhibition. Nat Cell Biol 2010;12(9):863-875.

14. Luciani A, Villella VR, Esposito $S$, et al. Cystic fibrosis: a disorder with defective autophagy. Autophagy 2011;7(1):104-106.

15. Abdulrahman BA, Khwk AA, Akhter A, et al. Depletion of the ubiquitin-binding adaptor molecule SQSTM1/p62 
from macrophages harboring cftr $\Delta F 508$ mutation improves the delivery of Burkholderia cenocepacia to the autophagic machinery. J Biol Chem 2013;288(3):20492058. doi: 10.1074/jbc.M112.411728.

16. Assani K, Tazi MF, Amer AO, Kopp BT. IFN- $\gamma$ stimulates autophagy-mediated clearance of Burkholderia cenocepacia in human cystic fibrosis macrophages. PLoS One 2014;9(5):e96681. doi: 10.1371/journal.pone.0096681

17. Junkins RD, Shen A, Rosen K, McCormick C, Lin TJ. Autophagy enhances bacterial clearance during P. aeruginosa lung infection. PLoS One 2013;8(8):e72263. doi: 10.1371/journal.pone.0072263.

18. Junkins RD, McCormick C, Lin TJ. The emerging potential of autophagy-based therapies in the treatment of cystic fibrosis lung infections. Autophagy 2014;10(3):538-547. doi: 10.4161/auto. 27750 .

19. Maurer K, Torres VJ, Cadwell K. Autophagy is a key tolerance mechanism during Staphylococcus aureus infection. Autophagy 2015;11(7):1184-1186. doi: 10.1080/15548627.2015.1058685.

20. Decreamer M, Janssens W, Miravitlles M. Chronic obstructive pulmonary disease. Lancet 2012;379(9823):13411351. doi: 10.1016/S0140-6736(11)60968-9.

21. Salvi SS, Barnes PJ. Chronic obstructive pulmonary disease in non-smokers. Lancet 2009;374(9691):733-743. doi: 10.1016/S0140-6736(09)61303-9.

22. Tetley TD. Inflammatory cells and chronic obstructive pulmonary disease. Curr Drug Targets Inflamm Allergy 2005;4(6):607-618.

23. Kirkham PA, Barnes PJ. Oxidative stress in COPD. Chest 2013;144(1):266-273. doi: 10.1378/chest.12-2664.

24. Demedts IK, Demoor T, Bracke KR, Joos GF, Brusselle GG. Role of apoptosis in the pathogenesis of COPD and pulmonary emphysema. Respir Res 2006;7(53):1-10.

25. Park JW, Ryter SW, Choi AM. Functional significance of apoptosis in chronic obstructive pulmonary disease. COPD 2007;4(4):347-353.

26. Yamada Y, Tomaru U, Ishizu A, et al. Decreased proteasomal function accelerates cigarette smokeinduced pulmonary emphysema in mice. Lab Invest 2015;95(6):625-634. doi: 10.1038/labinvest.2015.43.

27. Chillappagari S, Preuss J, Licht S, et al. Altered protease and antiprotease balance during a COPD exacerbation contributes to mucus obstruction. Respir Res 2015;16(85):1-9. doi: 10.1186/s12931-015-0247-x.

28. Chen ZH, Kim HP, Sciurba FC, et al. Egr-1 regulates autophagy in cigarette smoke-induced chronic obstructive pulmonary disease. PLoS One 2008;3(10):e3316. doi: 10.1371/journal.pone.0003316.

29. Kim HP, Wang X, Chen ZH, et al. Autophagic proteins regulate cigarette smoke-induced apoptosis: protective role of heme oxygenase-1. Autophagy 2008;4(7):887-895.

30. Chen ZH, Lam HC, Jin Y, et al. Autophagy protein microtubule-associated protein 1 light chain-3B (LC3B) activates extrinsic apoptosis during cigarette smoke-induced emphysema. Proc Natl Acad Sci U S A 2010;107(44):1888018885. doi: 10.1073/pnas.1005574107.

31. Tran I, Ji C, Ni I, Min T, Tang D, Vij N. Role of cigarette smoke-induced aggresome formation in Chronic Ob- structive Pulmonary Disease-Emphysema pathogenesis. Am J Respir Cell Mol Biol 2015;53(2):159-173. doi: 10.1165/rcmb.2014-01070C.

32. Monick MM, Powers LS, Walters K, et al. Identification of an autophagy defect in smokers' alveolar macrophages. J Immunol 2010;185(9):5425-5435. doi: 10.4049/jimmunol.1001603.

33. Taylor AE, Finney-Hayward TK, Quint JK, et al. Defective macrophage phagocytosis of bacteria in COPD. Eur Respir J 2010;35(5):1039-1047. doi: 10.1183/09031936.00036709.

34. Cloonan SM, Lam HC, Ryter SW, Choi AM. "Ciliophagy»: the consumption of cilia components by autophagy. Autophagy 2014;10(3):532-534. doi: 10.4161/auto.27641.

35. Lam HC, Cloonan SM, Bhashyam AR, et al. Histone deacetylase 6-mediated selective autophagy regulates COPD-associated cilia dysfunction. J Clin Invest 2013;123(12):5212-5230. doi: 10.1172/JCI69636.

36. Mariño G, Niso-Santano M, Baehrecke EH, Kroemer G. Self-consumption: the interplay of autophagy and apoptosis. Nat Rev Mol Cell Biol 2014;15(2):81-94. doi: 10.1038/nrm3735.

37. Farooq MB, Walsh GM. Autophagy and asthma. Clin Exp Allergy 2016;46(1):7-9. doi: 10.1111/cea.12633.

38. Jyothula SS, Eissa NT. Autophagy and role in asthma. Curr Opin Pulm Med 2013;19(1):30-35. doi: 10.1097/ MCP.0b013e32835b1150.

39. Martin LJ, Gupta J, Jyothula SS, et al. Functional variant in the autophagy related 5 gene promoter is associated with childhood asthma. PLoS One 2012;7(4):e33454. doi: 10.1371/journal.pone.0033454.

40. Poon AH, Chouiali F, Tse SM, et al. Genetic and histological evidence for autophagy in asthma pathogenesis. J Allergy Clin Immunol 2012;129(2):569-571. doi: 10.1016/j. jaci.2011.09.035.

41. Dickinson JD, Alevy Y, Malvin NP, et al. IL-13 activates autophagy to regulate secretion in airway epithelial cells. Autophagy 2016;12(2):397-409. doi: 10.1080/15548627.2015.1056967.

42. Ban GY, Pharm DL, Trinh THK, et al. Autophagy mechanisms in sputum and peripheral blood cells of patients with severe asthma: a new therapeutic target. Clin Exp Allergy 2016;46(1):48-59. doi: 10.1111/cea.12585.

43. Selman M, Pardo A. Revealing the pathogenic and aging-related mechanisms of the enigmatic idiopathic pulmonary fibrosis. an integral model. Am J Respir Crit Care Med 2014;189(10):1161-1172. doi: 10.1164/ rccm.201312-2221PP.

44. Leung J, Cho Y, Lockey RF, Kolliputi N. The role of aging in idiopathic pulmonary fibrosis. Lung 2015;193(4):605610. doi: 10.1007/s00408-015-9729-3.

45. Chilosi M, Carloni A, Rossi A, Poletti V. Premature lung aging and cellular senescence in the pathogenesis of idiopathic pulmonary fibrosis and COPD/emphysema. Transl Res 2013;162(3):156-173. doi: 10.1016/j. trsl.2013.06.004.

46. López-Otín C, Blasco MA, Partridge L, Serrano M, Kroemer G. The hallmarks of aging. Cell 2013;153(6):11941217. doi: 10.1016/j.cell.2013.05.039. 
47. Patel AS, Lin L, Geyer A, et al. Autophagy in idiopathic pulmonary fibrosis. PLoS One 2012;7(7):e41394. doi: 10.1371/journal.pone.0041394.

48. Araya J, Kojima J, Takasaka N, et al. Insufficient autophagy in idiopathic pulmonary fibrosis. Am J Physiol Lung Cell Mol Physiol 2013;304(1):L56-L69. doi: 10.1152/ ajplung.00213.2012.

49. Gui YS, Wang L, Tian X, et al. mTOR overactivation and compromised autophagy in the pathogenesis of pulmonary fibrosis. PLoS One 2015;10(9):e0138625. doi: 10.1371/journal.pone.0138625.

50. Parker MW, Rossi D, Peterson M, et al. Fibrotic extracellular matrix activates a profibrotic positive feedback loop. J Clin Invest 2014;124(4):1622-1635. doi: 10.1172/ JCI71386.

51. Nho RS, Hergert P. IPF fibroblasts are desensitized to type I collagen matrix-induced cell death by suppressing low autophagy via aberrant Akt/mTOR kinases. PLoS One 2014;9(4):e94616. doi: 10.1371/journal.pone.0094616.

52. Clarke DL, Carruthers AM, Mustelin T, Murray LA. Matrix regulation of idiopathic pulmonary fibrosis: the role of enzymes. Fibrogenesis Tissue Repair 2013;6(1):20. doi: 10.1186/1755-1536-6-20.
53. Sosulski ML, Gongora R, Danchuk S, Dong C, Luo F, Sanchez CG. Deregulation of selective autophagy during aging and pulmonary fibrosis: the role of TGF 1 . Aging Cell 2015;14(5):774-783. doi: 10.1111/acel.12357.

54. Bueno M, Lai YC, Romero Y, et al. PINK1 deficiency impairs mitochondrial homeostasis and promotes lung fibrosis. J Clin Invest 2015;125(2):521-538. doi: 10.1172/ $\mathrm{JCl} 74942$.

55. Cabrera S, Maciel M, Herrera I, et al. Essential role for the ATG4B protease and autophagy in bleomycin-induced pulmonary fibrosis. Autophagy 2015;11(4):670-684. doi: 10.1080/15548627.2015.1034409.

\section{$\triangle$ Correspondencia:}

Biól. Mariana Maciel Herrerías

Laboratorio de Bioquímica,

Departamento de Biología Celular.

Facultad de Ciencias, Universidad Nacional

Autónoma de México.

Correo electrónico: marianamaciel19@gmail.com

Los autores declaran no tener conflicto de intereses. 